\title{
GENERIC PRESCRIBING, BRAND AND GENERIC SUBSTITUTION, AVAILABILITY AND COST EFFECTIVENESS OF THE AVAILABLE MEDICINES
}

\author{
Khanal $\mathrm{DP}^{1^{*}}$, Adhakari $\mathrm{P}^{2}$, Chapagain $\mathrm{S}^{3}$, Rayamajhi $\mathrm{S}^{3}$, Nakarmi $\mathrm{S}^{3}$ and Adhakari $\mathrm{B}^{3}$.
}

${ }^{1,3}$ Department of Pharmacy, Manmohan memorial Institute of health sciences, Tribhuvan University, Soalteemode Kathmandu.

${ }^{2}$ Department of Pharmacy, Purbanchal University College of Medical and Allied Health Science, Goath Gaon, Biratnager, Nepal.

"Corresponding author:

Dharma Prasad Khanal, Professor and Head of Department, Department of Pharmacy, Manmohan memorial Institute of health sciences, Tribhuvan University, Soalteemode Kathmandu,Nepal, Email: drdpk@ mmihs.edu.np

\section{ABSTRACT}

Background: Pharmaceuticals play a key role in the prevention and treatment of disease. The worldwide availability of effective, safe and affordable pharmaceuticals is a key challenge for the global public health system. Large economic interests are at stake within the field of pharmaceuticals. Pharmaceutical sales are existed as trade in many part of developing world including Nepal. At the same time, consumers (patients) are at mercy and unable to judge the quality, safety and, in many cases, the efficacy of the medicine, as well as whether the price is reasonable or not. They depend on others for assured quality medicines. Objective of this study was to find out the prescription written in generic name, availability of generic medicine in hospital pharmacy and outside pharmacy and cost effectiveness of brand -generic substitution in Manmohan Memorial Hospital Premises, Shyombu Kathmandu.

Methods: All relevant information of brand-generic medicines and generic prescribing were recorded from the patient chart stored at medical record department except orthopedic department.In Orthopedic department, OPD prescriptions were recorded. Hospital pharmacy and outside pharmacy have been audited for the availability of generic medicines. Face to face interview with pharmacists in hospital and Drug Retailers were taken regarding the medicine available in generic name and medicine substitution. The recorded data were analyzed.

Results: Only 298 out of 5120 medicines were found in generic name mostly from medical department, 215 medicines out of 812 medicines. There was no any single generic prescription in orthopedic department. In the hospital pharmacy and outside pharmacy have very few generic medicines. So no question of brand substitution by generic here. Mostly some large volume parental were available in generic names.

Conclusion: Only $5.82 \%$ of medicines were prescribed in generic name. Concerns over the therapeutic equivalence of branded products and generics are common amongst physicians, pharmacists and industries of course.

Key Words: Generic prescribing, Generic medicine, Brand medicine, Prescription, 


\section{INTRODUCTION}

Generic medicines are defined according to Austrian drug law/the Medicinal Products Act as well as by EU Directive, is that a generic medicinal product 'is a product which has the same qualitative', i.e. kind of active substance, 'and quantitative', i.e. amount of active substance, 'composition as the reference medicinal product'. For the sake of simplicity, the reference product is often also referred to as the originator ${ }^{1}$.

We have to understand that generic drugs are bioequivalent replicas of brand name drugs, containing the same active ingredients and with identical quality, safety, and efficacy profiles. Any differences are limited to inactive ingredients, like fillers, coloring, flavoring, and stabilizing agents. Generics can, in theory, be sold for a fraction of the price of brand name drugs for two reasons. First, it is relatively cheap to bring a bioequivalent product to market. Second, the market for the drug typically already exists, significantly reducing marketing expenses. But Bioavailability or bioequivalence tests need to be conducted in order to demonstrate the equivalence between the generic medicinal product and the reference brand (innovator's brand) product.

Generic medicine is one of the most commonly used pharmaceutical cost-containment strategies, aimed at stimulating competition on the off-patent market and thereby reducing costs without compromising quality and efficacy.Use of generic medicines significantly reduces the cost of medicines to both governments and patients in the developed world. Generic medicines are those produced without a license from the innovator company when the patent or other market exclusivity rights on the innovator product has expired ${ }^{2}$.

Generic medicines face the same regulatory path way as their brand counter and contain the same active pharmaceutical ingredient (API) butmay use different inactive ingredients like fillers, colors etc that are approved by the National Drug Control Authority (In Nepal Department of Drug Administration). In principal generic medicines deliver the same amount of active ingredient to the body in the same way as the original brand. All quality studies applied to the original brand must also be performed on the generic medicine. Some human trials testing safety and performance from the starting of innovation do not have to be repeated because we already know the outcomes of these tests, and the Department of Drug Administration accepts that the same safety and performance outcomes will be shared by the generic medicine and original brand. A generic medicine must be "bioequivalent" to the original brand. Meeting these requirements requires rigorous scientific testing and is harder than it seems to manufacturers and importers-suppliers. Should the generic medicine fail to meet the standards, it will not be approved for sale in the territory of Nepal; this only can assure the quality and efficacy as well as the open substitution of brands with generics. Approval of a generic medicine is based on the demonstration of interchangeability or therapeutic equivalence to the innovator through bioequivalence studies. Bioequivalence is the absence of a significant difference in the rate and extent to which the active ingredient or active moiety in pharmaceutical equivalents or pharmaceutical alternatives becomes available at the site of drug action when administered at the same dose ${ }^{3}$.

Each year, Americans save an estimated $\$ 8$ billion to $\$ 10$ billion at retail pharmacies by purchasing generic drugs rather than brand-name medications, the US Food and Drug 
Administration reports. The lower cost, of course, is the key advantage of generics. But the very reason for the cost savings - the fact that generic drugs do not have to undergo the large, expensive clinical trials that are required for approval of brand-name medications-gives rise to questions about the quality and safety of generics. Are these concerns justified? Under what circumstances is it safe to prescribe generics, or to substitute a generic for a brand-name drug? Are brand-name drugs always better?

To answer these questions researchers conducted a thorough evidence review, which included numerous randomized controlled trials (RCTs) and case reports, as well as a single meta-analysis that assessed the benefits and risks of generics. Generics: On the positive side safety and efficacy. They found little evidence that generic drugs are less safe or less effective than their brand-name equivalents. The meta-analysis, for example, 2 included 47 studies (38 of 47 were RCTs) covering 9 subclasses of cardiovascular medications. In trials involving beta-blockers, diuretics, calcium channel blockers, antiplatelet agents, statins, angiotensin-converting enzyme inhibitors, and alpha-blockers, no evidence of superiority of brand-name drugs vs generics was found. Generic drugs typically cost $30 \%$ to $60 \%$ less than their brand-name counterparts, and widespread use of generics has the potential to reduce the price of other brand-name drugs by creating more competition ${ }^{3}$.

Avika Dixit, Neeta Kumar and Sanjiv Kumar in India ${ }^{4}$ found that Generic medicines are as effective as branded medicines. The initiative of the government and Medical Council of India by making it mandatory for doctors to write generic prescriptions has raised many concerns related to generic drugs manufacturing, availability and quality. Experience in the USA and Canada support the argument in favor of generic medicine. WHO state that generic medicines can enable huge cost-savings as they create competition, driving down prices? In medicines regulation and in WHO prequalification, the efficacy of generics is demonstrated by bioequivalence studies. Registration of generic products and generic substitution policies are well advanced in high income countries, but are still under development in low- and middleincome countries. WHO is providing norms and standards for medicines quality assurance in member States, including resource-constrained ones? Nonetheless, in many countries the demonstration of interchangeability remains non-existent or is not fully enforcedby law ${ }^{5-6 .}$

Likewise, most of the pharmaceutical manufacturers in our country, Nepal are inexperienced in performing bioequivalence studies to the required regulatory standard. Here is the main problem and resistance of the manufacturers. If the Bioequivalence assessment is routine practice of our manufacturers in that case there will be no question of interchangeability. Now Nepal government has to pay attention to establish the national centre for bioequivalence and it should be mandatory for all generic products that totally eliminate the low quality issues. WHO (Ibid) stated that the pivotal in supporting generic prescribing and substitution policies, which are important to increasing access to medicines. However, these findings cannot necessarily be extrapolated to other nationally approved products, especially in resource-constrained settings. National Drug Control Authority should ensure that generic products are interchangeable before granting approval. The WHO estimated that at least $30 \%$ of the world's population lacks regular access to essential medicines, and the figure increases to $50 \%$ in the poorest countries of Africa and Asia. Generic medicines have been available in Australia for 40 years, and widely supplied 
through the Pharmaceutical Benefits Scheme for over 20 years, delivering the same health benefits as the original brands at a lower cost to government and the community ${ }^{7}$.

Sonali P Suryawanshi et $\mathrm{al}^{8}$ concluded that branded generic cephalosporins, equieffective as the branded versions, with lesser cost to the patient and can be used clinically. However, cost benefit to the patients, being hyped in the media, is very meager compared to the profit margins for the retailers. In India, many pharmaceutical companies manufacture a product under both types, i.e., the branded product which they advertise and push through doctors and branded-generics which they expect to be sold over the counter by retailers. The researcher found that the price difference being only $71-100 \%$ of the branded formulation.

In Sweden generic substitution was mandatory and implemented in 2002. The reform has proven effective, and today Sweden has among the lowest prices of off-patent medicines in Europe. However, generic substitution is still debated, as it is found to worry and confuse patients and thus pose a risk to patient safety. More than $56 \%$ of prescriptions of dispensed medicines in Europe are generic yet they account for just $22 \%$ of the total expenditure on medicines. Generic medicine pricing in Europe is influenced by price competition from Indian generic medicine manufacturers and by domestic policy towards generic medicines. An international comparison of generic medicine prices demonstrated that prices of generic medicines in India are substantially lower than in Europe. Two studies found that generic medicine discounts ranged from 20 to $70 \%$ off the wholesaler selling price in France and maximum discounts exceeded $50 \%$ of the Drug Tariff price in the $\mathrm{UK}^{9}$. The proportion of prescriptions filled with generics ranges from $17 \%$ in Switzerland to $83 \%$ in the United Kingdom. By comparison, the United States has historically had low generic drug prices and high rates of generic drug use $(84 \%$ in 2013), but has in recent years experienced sharp price increases for some offpatent products. The prices and market shares of generics vary widely across Europe. For example, prices charged by manufacturers in Switzerland are, on average, more than 2.5 times those in Germany and more than 6 times those in the United Kingdom, based on the results of a commonly used price index.

A study in the US found that while the price of a first entry generic medicine was only marginally lower than the innovator brand price, the entry of a second generic medicine dropped the average generic price to about $50 \%$ of the innovator brand price. When a large number of generic medicines entered the market, the average generic price fell to $20 \%$ or even lower. The introduction of mandatory generic substitution policy in Sweden resulted in a $15 \%$ reduction in overall medicine prices and more than $40 \%$ decrease in prices of off-patent medicines within 4 years of the policy implementation ${ }^{10}$. Similar results were found in Finland, with a $10.6 \%$ reduction in substitutable medicine prices and up to $80 \%$ price decrease for some medicines during the first year of mandatory generic substitution policy implementation. Covering 36 countries, including the 27 European Union member states, Teva is Europe's biggest provider of tried-and-trusted generic medicines. Every single minute of every day, approximately 2,500 Teva packs are dispensed in the EU. Teva Europe estimates that we save some $€ 8$ billion each year from Europe's healthcare budget by supplying cost-effective treatments ${ }^{11-13}$.

In UK, there is considerable interest and debate concerning the place of generic substitution (switching from a brand to generic product); and on therapeutic substitution, that is, switching to a cheaper, but apparently equivalent, product, usually within the drug class. Generic substitution by pharmacists is standard practice in UK hospital settings, and is being proposed for 
implementation in primary care ${ }^{14-16}$. Although most prescriptions are already written generically (83\% in the community in England in 2008), there are still cost savings that could be made if generic medicines are substituted against prescriptions written by branded name or by getting prescribers to adhere to advice to prescribe generically ${ }^{17-20}$. Assuming the trend for growth in prescribing overall continues, and that generic prescribing and dispensing increases to 90 per cent, all other things being equal, by 2023/4. With generic prescribing rates averaging around 84 per cent, further improvements may be unlikely. However, a proportion of medicines, although prescribed generically, are still dispensed as proprietary; on average these medicines cost nearly seven times more than those prescribed and dispensed generically ${ }^{21}$.

In 2005, some 50 percent of all prescriptions dispensed in the United States were unbranded generic medications. Until 2016, the percentage increased to 84.6 percent $^{22}$. The high cost of some medicines in India has made the treatment of many common and uncommon diseases unaffordable to the poor and a strain on the budgets of even middle-class citizens. The authors found that branded generics in terms of retailer purchase price versus maximum retail price (MRP) was 25\%-30\%; for the nominally branded generics, this mark-up was far higher at 201\%-1016\%, giving the retailer (but not the company) an enormous margin of profit. The MRP of branded generics was $0 \%-41 \%$ higher than that of the nominally branded generics ${ }^{23}$. More than $80 \%$ of prescribed medicines, both in the USA and the UK, are now dispensed as "generics". The attraction of generic prescribing is financial. Generic products are usually a fraction of the cost of their branded equivalents and thus save money for payers whether they are individuals, health-insurance companies, or publicly funded health-care systems ${ }^{24}$.

Even with those having narrow therapeutic index drugs such as antiarrhythmic agents and warfarin show $100 \%$ clinical equivalence ${ }^{25}$. The Government of Bangladesh controls the price of 209 generic drugs in specified dosage forms, along with 41 active pharmaceutical ingredients produced in the country and medicine imports ${ }^{26-27}$. Nonetheless, irrespective of the level of healthcare delivery system, prescribing drugs by generic name reduces duplicity, improves access to medicines, and subsequently, escalates patient compliance with drug therapy and disease control. It is expected that emphasizing to medical students about generic medicines will improve and promote rational prescribing ${ }^{28}$. Lack of official channels to announce the patent status of major drugs. This is one potential gap which the media, for the public or professional alike, could help disseminate such information and Lack of commercial incentive to switch to cheaper generic drugs. Innovator or patented drugs often cost more \& are thus sold at higher prices. It is the responsibility of drug control authority and manufacturer to ensure that generics to be marketed are not just chemically equivalent, but also biologically equivalent (or bioequivalent) in the drug's effect within the human body, usually to an acceptable limit (within $+/-20 \%$ for most medicines). With such a stringent regulation in Singapore, there are no strong reasons to believe that innovators are more efficacious or safer compared to generic medicines. So, the first requirement for a successful generics policy is a mechanism sufficient to provide certainty that generic products are of assured quality. Clearly, this involves having an effective regulatory system (and an effective enforcement of anti-counterfeiting policies). Assured quality of generic medicines is the pre-condition for all measures to take effect ${ }^{29}$.

In Pakistan, during the period in which medicines were required to be manufactured and marketed by the INN and government mounted a campaign to induce doctors to write the 
manufacturer's name along with the generic name on prescriptions. Doctors, who had expressed concerns about unqualified dispensers and/or sellers of medicines making inappropriate generic substitutions, were responsive to this suggestion. In an early publication, a survey of doctors conducted by a leading manufacturer indicated that about $60 \%$ of the prescriptions they had written included a specific company name. Amongst the Sub-Saharan countries, policies allowing generic substitution exist in Ghana, Uganda and South Africa. In the latter, the system introduced in 2003 provides for a mandatory offer of generic substitution. In its current form, the South African generic substitution provision compels pharmacists to offer patients a generic substitute for any medicine prescribed, unless the prescriber explicitly states that the medicine should not be substituted or where the price of the generic is higher than that of the originator. A mandatory generic substitution policy in the public sector exists in Indonesia. This was done in conjunction with an industrial policy of production of "logo generic medicines" led by stateowned manufacturers and with the entry of private manufacturers being encouraged by the government $^{30}$.

In various Latin American countries, the substitution of medicines has been restricted to a certain list of medicines (i.e., those for which therapeutic equivalence is proven) ${ }^{30}$. As of December 2012, out of a total of 29 European countries (the then 27 EU Member States, plus Croatia and Norway), generic substitution is not allowed in seven countries (i.e., Austria, Bulgaria, the private sector in Cyprus, Greece, Ireland, Luxembourg and the UK). It is optional in 16 countries, and is mandatory in five countries (i.e., Denmark, Finland, Germany, the public sector in Malta and Sweden) ${ }^{31}$.

\section{MATERIALS AND METHODS}

All relevant information of brand-generic medicines and generic prescribing were recorded from the patient chart stored at medical record department except orthopedic department. In Orthopedic department, OPD prescriptions were recorded. Hospital pharmacy and outside pharmacy have been audited for the availability of generic medicines. Face to face interview with pharmacists in hospital and Drug Retailers were taken regarding the medicine available in generic name and medicine substitution. The recorded data were analyzed.

Inclusion Criteria: The following six illnesses (UTI, Diabetes, Appendicitis, COPD, Cholelithiasis and Gastroenteritis) were randomly selected for monitoring generic prescribing. In Orthopedic OPD all prescription were recorded for a week during July 2017.

Ethical clearance: Approved by institutional review committee (IRC) of Manmohan memorial Institute of Health Sciences.

Study period:Patient charts archived at the medical record department during the years 2015 and 2016 were reviewed to find out the generic prescription in case of Diabetes, Appendicitis, COPD and Gastroenteritis. For UTI prescription of June, July and August 2018 were reviewed and recorded.

\section{RESULTS AND DISCUSSION}

In total 5120 medicine items from 1276 prescriptions(UTI 200, Diabetes 71, Appendicitis 210, COPD 285, Cholelithiasis 200, orthopedic 110 and Gastroenteritis 200), were analyzed from 
surgery, medicine and Orthopedic departments for generic prescription. Only 298 (5.82\%) medicines were found in generic name mostly from medicine department for UTI 215 items out of $812(26.5 \%)$ prescribed in generic name. In 2016, the percentage of generic prescription in USA was 84.6 percent ${ }^{22}$. One multicentre study in eight cities of South and Midwest of Brazil reported that most drugs $(86.1 \%)$ were prescribed by generic name ${ }^{28}$. In The Bangladesh, court ordered that 'doctors must use generic drug names rather than specifying particular brands. The Bangladesh pharmaceutical industry produces about 8000 generic drugs under 26,813 registered brands in different dosages and strengths ${ }^{26-27}$. In Austria, the number of all generics prescriptions has more than doubled from $11 \%$ in 2000 to $23 \%$ in $2010^{5-6}$. In Indonesia, once legislation on generic prescribing is enacted, one of its critical tests is the rate at which doctors prescribe by INN only. A positive perception in terms of quality, efficacy and cost savings is the key driver to increase generic prescription rates. More than $56 \%$ of prescriptions of dispensed medicines in Europe are generic ${ }^{29}$. In Nepal all government hospitals have the procurement list in generic version but doctors are writing the prescription in brands ${ }^{31}$.

\section{Generic prescribing}

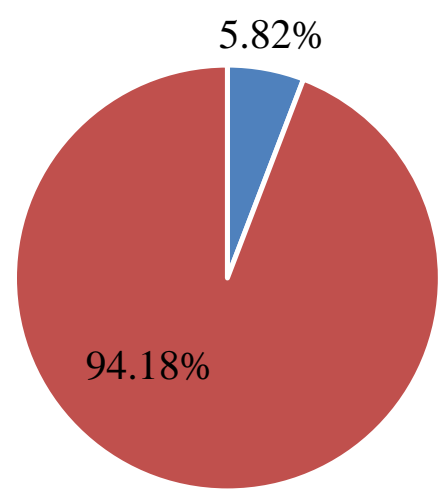

- Drugs prescribed in generic name - Drugs not prescribed in generic name

\section{Figure1: Percentage of generic drug prescribing in study setting.}

Practice of the Europe and America showed that generic medicines can provide substantial savings to healthcare systems. However, there are many challenges in implementing fully generic medicine policies to get the maximum benefits. An immediate priority is to convince more physicians, pharmacists, and patients that generic drugs are bioequivalent to branded products, although this may take time. The main attention to be paid on efficacy, safety and scientific data base substitution. Meanwhile, management of bioequivalence study and much could be achieved by requiring generic prescribing and substitution where such policies are not yet in place in our country Nepal. If industry is regulated into noncompetitiveness and nonprofitability, it will implode; interest and investment in the industry will diminish, leaving behind a market that is bereft of quality because such is all that can survive the contraction of profit margins $3,7,14,18,30$ and 31 . 


\section{CONCLUSION}

Only $5.82 \%$ of medicines were prescribed in generic name. Generic medicines are not available in the market. Good quality control and regulation to maintain therapeutic equivalence is prerequisite for generic prescribing and dispensing. Concerns over the therapeutic equivalence of branded products and generics are common amongst physicians, pharmacists and industries of course. In Nepal, already has a pharmaceutical environment that is strongly dominated by generic branded medicines (mostly patent off) rather than by brands. So there is no question of substitution and costeffectiveness. There is no question of substitution as generic medicines in real sense are not available in the hospital pharmacy and outside pharmacy.

\section{RECOMENDATION}

Nationwide auditing is necessary to find out the present situation of generic medicine supply and prescribing. Present study cannot be generalized. Education programs to physician, pharmacists and drug retailers from drug regulatory body and ministry of health is necessary. Promulgation of government law regarding generic medicine manufacturing and generic prescribing is necessary for the cost effective supply and availability of essential medicines to general public.

\section{REFERENCES}

1. Official Journal of the European Union, L 311, 28.11, 2001, p. 67.

2. Rathe J, Sondergaard, J., Jarbol, D., Hallas, J., Andersen M. Patients'concerns about their medicine after a generic switch: a combined cross-sectional questionnaire and registrer study. Pharmacoepidemol Drug Saf.2014; 23: 965-973.

3. Lewek P, The Journal of family practice.2010; 59(11):634-640,.

4. Dixit A, Kumar N ,Kumar S, Research Gate.2018;20(1):84-90,

5. WHO, Generic medicines, WHO Drug Information Vol. 30, No. 3, WHO, 2011.

6. World Health Organization (WHO), 2011. The world medicines situation: Access to essential medicines as part of the right to health. In: Hogerzeil, H. V. \& Mirza, Z. (eds.). Geneva: World Health Organization, 2016.

7. Generic Medicines - Facts and Fallacies-Generic and Biosimilar Medicine Association https://www.guild.org.au/_ data/assets/pdf_ file/0025/5848/ generic-medicines- factsand-fallacies-.pdf, retrieve on 2nd September 2018.

8. Suryawanshi SP, Totlani PS, Sahasrabudhe RA. Branded Versus Generic (BrandedGeneric) Medicines-For Whose Benefit?. J Basic Clin Pharm. 2017; 8:158-161

9. Simones S. Journal of Medical Economics. 2008,11(1):

10. Pharmaceutical Benefits Board (LFN), 2007.

11. http://www.tevapharm.com/teva_worldwide/europe/ retrieve on $2^{\text {nd }}$ September 2018.

12. Mythbusters - Myth: Generic drugs are lower quality and less safe than brand name drugs. http://apps.who.int/medicinedocs/documents/s20959en/s20959en.pdf Eurohealth Vol 14 No 4, 2007.

13. https://www.thepharmaletter.com/article/generic-drugs-save-european-100-billion-eurosper-year. The Pharma letter, 2016.

14. Dylst P, Vulto A, Simoens S. Overcoming challenges in market access of generic medicines in the European Union. Journal of Generic Medicines. 2012; 9:21-28. 
15. Olivier J. Wouters, Panos G. Kanavos, A comparison of generic drug prices in seven European countries: a methodological analysis. BMC Health Services Research. https://doi.org/10.1186/s12913-017-2184-5, 2017.

16. Oliviler J. Wouters, Panos G. Kanavos, and Martin McKee, Comparing Generic Drug Markets in Europe and the United States: Prices, Volumes, and Spending. Milbank Q. 2017.

17. The US Food and Drug Authority (FDA), 2010, the US Food and Drug Authority (FDA), 2010. Generic competition and drug prices [Online]. Available: http://www.fda.gov/AboutFDA /Centers Offices/Officeo Medical Products and Tobacco/CDER/ucm129385.htm (accessed 202 2013), Retrieve on 2nd September 2018.

18. Pharmaceutical Benefits Board (LFN), 2007, Pharmaceutical Benefits Board (LFN), 2007. The swedish pharmaceutical reimbursement system - a brief overview [Online]. http://www.tlv.se/ Upload/ English/ ENG -swe-pharma -reimbursementsystem.pdf (accessed 1001 2013).

19. Setälä A, The impact of generic substitution on price competition in Finland, Eur. J. Health Econ. 2008; 9:185-191.

20. Duerden MG, Hughes DA. Generic and therapeutic substitutions in the UK: are they a good thing? Br J Clin Pharmacol. 2010; 70(3): 335-341.

21. https://www.kingsfund.org.uk/blog/how-much-has-generic-prescribing-and-dispensingsaved-nhs, 2015, Retrieve on 2nd September 2018.

22. Branded vs. generic U.S. drug prescriptions dispensed 2005-2016. Stastista, https://www.statista.com/statistics/205042/proportion-of-brand-to-generic-prescriptionsdispensed/, Retrieve on 2nd September 2018.

23. Chittaranjan Andrade, T. S. Sathyanarayana Rao. Prescription writing: Generic or brand? Indian J Psychiatry. 2017;59(2): 133-137.

24. Michael Rawlins, The Lancet, ohttps://www.thelancet.com /journals/lancet/article/ PIIS0140-6736(15)60044-7/fulltext, 2015.

25. Kesselheim AS, Misono AS, Lee JL, Stedman MR, Brookhart MA, Choudhry NK, et al. Clinical equivalence of generic and brand-name drugs used in cardiovascular disease: A systematic review and meta-analysis. JAMA. 2008;300:(25):14-26.

26. Haque M - Generic medicine and prescribing: A quick assessment. Advances in Human Biology. 2017; 7(3): 101-108.

27. Dylst P, Simoens S; Does the market share of generic medicines influence the price level?: a European Pharmacoeconomics.2011,10:875-882. doi:10.2165/11585970000000000-00000, 2011.

28. FerreiraI MB, Heineck I, Flores LM, Aline Camargo L, Pizzol TS, Torresm IL, et al. Rational use of medicines: Prescribing indicators at different levels of health care. Braz J Pharm Sci.2013; 49:329-340.

29. https://www.pss.org.sg/know-your-medicines/commonly-asked-questions/generic-drugcost\#.W4qs0CQzbIU. Retrieve on $2^{\text {nd }}$ September 2018.

30. Health Action International (HAI)- Policy Options for Promoting the Use of Generic Medicines in Lowand Middle-income Countries, March 2016. 
31. Vogler $S$, The impact of pharmaceutical pricing and reimbursement policies on generics uptake: implementation of policy options on generics in 29 European countries-an overview. GaBI Journal. 2012;1(2): 93-100.

32. Procurement list of Tribhuvan University Teaching Hospital Maharajgung Kathmandu, Kanti Children Hospital Maharajgung Kathmandu, Hospital Mental Hospital Lagenkhel Lalitpur and Civil service Hospital Minbhawan, Kathmandu. 